\title{
Gesamtwirtschaftliche Wirkungen der Einführung eines gesetzlichen Mindestlohnes in Deutschland
}

In Deutschland ist „Armut trotz Arbeit“ selbst für Vollzeitbeschäftigte ein großes und weiter zunehmendes gesellschaftspolitisches Problem. Die Verhandlungsmacht der Gewerkschaften reicht in vielen Branchen nicht mehr aus, um diese Entwicklung allein auf tarifpolitischem Wege zu stoppen. Vor diesem Hintergrund mehren sich in der öffentlichen Diskussion die Stimmen, die für die Einführung eines gesetzlichen Mindestlohnes auch in Deutschland votieren. Die Gewerkschaft ver.di hat ein Konzept für die Einführung von Mindestlöhnen vorgelegt. Dessen Auswirkungen wurden mit Hilfe einer makroökonometrischen Simulation geprüft. Der Beitrag stellt die zentralen Ergebnisse dieser Simulationsrechnung dar.

\section{Einleitung}

In Deutschland arbeiten mittlerweile allein 2,7 Mio. Vollzeitbeschäftigte für einen Nettolohn unterhalb der Pfändungsfreigrenze für Alleinstehende (Bispinck/Schäfer 2006, S. 294). Etwa 6,9 Mio. Voll- und Teilzeitbeschäftigte erhalten einen Stundenlohn, der die Niedriglohnschwelle von $66 \%$ des Medianeinkommens unterschreitet (Jaehrling et al. 2006). Und die Zahl der von Einkommensarmut Betroffenen steigt seit Mitte der 1990er Jahre an.

Die Verhandlungsmacht der Gewerkschaften ist in vielen Branchen nicht ausreichend, um diese Entwicklung allein auf tarifpolitischem Wege zu stoppen. Dies hängt mit der langjährigen schlechten Arbeitsmarktlage, dem gesunkenen Organisationsgrad der Beschäftigten, aber auch mit der zunehmenden Erosion des Flächentarifvertrages zusammen. Zudem geht seit 2005 von den Regelungen des sogenannten Hartz IV-Gesetzes ein erheblich verstärkter Druck auf Arbeitslose aus, jede noch so schlecht bezahlte Arbeit anzunehmen.

Vor diesem Hintergrund werden in der öffentlichen wirtschaftspolitischen Diskussion die Stimmen immer zahlreicher, die für die Einführung eines allgemein verbindlichen gesetzlichen Mindestlohnes (GML) auch in Deutschland votieren. Dadurch soll dem Lohnbildungsprozess eine gesetzliche Untergrenze auf einem fest definierten Niveau gesetzt werden, welches die Reproduktion der Arbeitskraft im Regelfall ohne zusätzliche staatliche Unterstützung erlaubt.
Mittels der hier dokumentierten makroökonometrischen Studie soll ex ante simuliert werden, welche wesentlichen gesamtwirtschaftlichen Effekte kurz-, mittelund langfristig bei Umsetzung der von der Dienstleistungsgewerkschaft ver.di vorgeschlagenen Konzeption ${ }^{1}$ für die Einführung eines Mindestlohnes zu erwarten wären.

\section{Beschäftigungswirkungen eines gesetzlichen Mindestlohns}

\subsection{THEORETISCHE ANNAHMEN}

Im Zentrum der kontroversen theoretisch und empirisch geführten wissenschaftlichen Diskussion über die Effekte eines gesetzlichen Mindestlohns steht letztlich immer die Frage nach seinen Auswirkungen auf das Beschäftigungsniveau: Ist ein in bester sozialpolitischer Absicht eingeführter Mindestlohn tatsächlich geeignet, die Zahl der „working poor“ zu vermindern, ohne zugleich die Zahl der „jobless poor“ zu erhöhen?

Die Theorie gibt hier keine eindeutige Antwort. Während die Vertreter der neoklassischen Arbeitsmarkttheorie im Regelfall von erheblichen Beschäftigungsverlusten ausgehen (Stigler 1946; Ragnitz/Thum 2007), sind auch im Rahmen des neoklassischen Paradigmas unter der Annahme einer quasimonopsonistischen Arbeitsnachfrageseite grundsätzlich positive Beschäftigungseffekte durch die Einführung eines GML denkbar (Bhaskar/To 1999; Herr 2002; IMK 2006).
Auch vom effizienzlohntheoretischen Ansatz ausgehend kann die Erhöhung von Löhnen, etwa im Zuge der Einführung von Mindestlöhnen, beschäftigungsneutral sein oder auch beschäftigungsfördernde Wirkungen entfalten, da Lohnerhöhungen aus dieser partialanalytischen Sicht durchaus produktivitätssteigernd wirken können (Mankiw 1996, S. 167ff.).

Aus der keynesianischen Perspektive, die Kreislaufzusammenhängen Rechnung trägt, bestimmt in der Tendenz die Güternachfrage das realisierte Angebot und damit zugleich auch wesentlich das von den Unternehmen nachgefragte Arbeitsvolumen (Kromphardt 1987, S. 64ff.). Dagegegen wird in der einfachen neoklassischen Theorie von der Geltung des „Say'schen Gesetzes" ausgegangen, nach dem sich jedes Angebot seine Nachfrage schafft. Die Nachfrage ist somit hier die abhängige Variable (Kromphardt 1987, S. 75ff.). Für aus Veränderungen des Reallohnes erwachsende Variationen der Nachfrage und ihre Rückwirkungen auf das Angebot respektive den Output ist im Rahmen des neoklassischen Deutungsmusters also kein Platz.

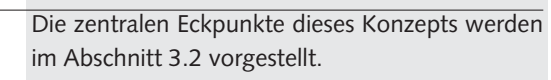

Bartsch Klaus, Dipl.-Ökonom, freiberuflicher Wirtschaftswissenschaftler, Geschäftsführer von KBE Klaus Bartsch Econometrics. Arbeitsschwerpunkte: Ökonometrische Prognose- und Simulationsmodelle und modellgestützte empirische Wirtschaftsforschung. e-mail: Bartscheconometrics@gmx.de 
Im Unterschied dazu wird im keynesianischen Ansatz der Doppelcharakter der Löhne berücksichtigt, welche grundsätzlich gleichzeitig sowohl betriebliche Kosten als auch gesamtwirtschaftliche Güternachfrage darstellen (Herr 2002, S. 4).

Vor diesem Hintergrund lässt insbesondere eine Ausweitung der Reallöhne ${ }^{2}$ von Niedriglöhnern durch die Einführung eines Mindestlohnes nennenswerte Nachfragewirkungen erwarten, da die Verwendungsstruktur der Einkommen dieser Personengruppe durch eine überdurchschnittlich hohe Konsumquote geprägt ist.

Bei dynamischer Betrachtung könnte dann zusätzlich die beobachtbare Tendenz zur Ausweitung von deflationär wirkendem Lohndumping gebremst werden, die sich aus der Massenarbeitslosigkeit und der daher insgesamt verschlechterten Verhandlungsposition der Gewerkschaften speist (Herr 2002, S. 4; IMK 2006, S. 31).

Ist die makroökonomische empirische Arbeitsnachfragefunktion der Unternehmen in Bezug auf die Entwicklung der realen Güternachfrage eher elastisch und im Hinblick auf das Reallohnniveau eher unelastisch, so wären im Ergebnis der Einführung eines Mindestlohnes insgesamt Beschäftigungsgewinne zu erwarten (IMK 2006, S. 30).

\subsection{EMPIRISCHE BEFUNDE}

Welcher Ansatz die realen Verhältnisse in der Tendenz jeweils am treffendsten abbildet, lässt sich letztlich nur auf der Basis empirischer Ergebnisse klären.

Für viele Länder mit Mindestlöhnen liegen zahlreiche empirische, meist mikrooder mesoökonometrisch angelegte Studien zu den Beschäftigungseffekten nach Einführung bzw. Erhöhung von gesetzlichen Mindestlöhnen vor ${ }^{3}$ (insbesondere die Synopsen bei OECD 1998; Ragacs 2003; Neumark/Wascher 2007), deren Auswertung Hinweise auf die zu erwartende Entwicklungstendenz bei der Beschäftigung im Niedriglohnbereich nach Einführung des GML in Deutschland geben kann.

Allerdings: Die empirischen Befunde zeigen keine eindeutige Tendenz. Jedoch überwiegen gerade für Länder, in denen die Qualifikations- und Altersstruktur der Niedriglöhner mit jener in Deutschland vergleichbar ist, Studien, die zum Ergebnis weitgehend fehlender oder aber leicht positiver Beschäftigungseffekte gelangen
(OECD 1998, Annex 2.B; Ragacs 2003; Schulten 2006a).

Daher erscheint es vertretbar, im Rahmen dieser Simulationsstudie von der Annahme auszugehen, dass die Einführung eines GML im Niedriglohnsektor beschäftigungsneutral wirkt, insoweit die prognostizierte Relation des Mindestlohnniveaus zum Durchschnittslohn („Kaitz-Index“) in der Bandbreite der Werte für jene Länder liegt, für die empirische Untersuchungen vorliegen.

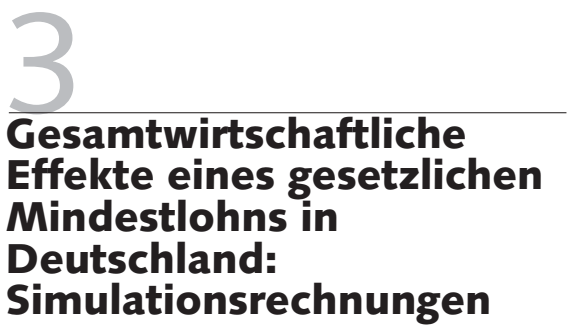

Das folgende Simulationsmodell geht davon aus, dass in Deutschland ab 2008 ein gesetzlicher Mindestlohn eingeführt wird. Das Modell orientiert sich an dem Mindestlohnkonzept der Gewerkschaft ver.di. Der Simulationszeitraum umfasst die Jahre 2008 bis 2020 .

\subsection{ZUR METHODIK DER UNTERSUCHUNG}

Der im Rahmen dieser makroökonometrischen Ex-Ante-Simulationsstudie gewählte Ansatz knüpft an das keynesianische Erklärungsmuster an. Dabei ergeben sich die Schätzungen für die prognoserelevanten zeitvariablen empirischen Lohn- und Nachfrageelastizitäten der Beschäftigung implizit aus der regressionsanalytisch ermittelten Parameterstruktur des Modells.

Die Simulationen wurden mit einem eigenen, fortlaufend gepflegten und weiterentwickelten makroökonometrischen Modell für die Bundesrepublik Deutschland mit aggregiertem Europamodul LAPROSIM (Langfristprognose- und Simulationsmodell) durchgeführt. ${ }^{4}$

Auf das Basisszenario aufsetzend wird das jeweilige Alternativszenario modelliert. Letzteres unterscheidet sich vom Basisszenario nur durch die Einführung eines gesetzlichen Mindestlohnes in der ,virtuellen Ökonomie" des Modells. Alsdann werden das Basisszenario und das GML-Szenario über geeignete numerische Spezifikationen in den Kontext des ökonometrischen Modells eingebracht, um sie „rechenbar“ zu machen. Anschließend wird das Modell auf der Basis der verschiedenen Szenariospezifikationen gelöst. Auf der Grundlage der Lösungen können dann für die Modellvariablen relevante absolute oder prozentuale Differenzen der Alternativlösung gegenüber der Basislösung berechnet werden.

\subsection{DIE SZENARIEN}

\section{REFERENZSZENARIO}

In das Referenzszenario (auch: Basisszenario) gehen neben den derzeit absehbaren Grundlinien der Entwicklung zentraler weltwirtschaftlicher und demografischer Parameter vor allem die Kernelemente der aktuellen wirtschafts- und fiskalpolitischen Beschlusslage und deren Fortschreibung $a$ priori ein. Da die hier vorgelegte Simulationsstudie die Effekte der Mindestlohnvarianten als Abweichungen von der Basislösung ausweist und diese Abweichungen aufgrund der nur schwach ausgeprägten Nichtlinearität des Modells auf kleine Veränderungen des Niveaus der Basislösungen nur unwesentlich reagieren, kann an dieser Stelle auf eine explizite Darstellung des Szenarios verzichtet werden.

\section{SZENARIO: GESETZLICHER MINDESTLOHN (VER.DI-KONZEPT)}

In der Mindestlohnvariante wird von der Einführung eines allgemeingültigen gesetzlichen Mindeststundenlohnes (GML) in Höhe von 7,50€ ab 1.1.2008 ausgegangen. Dabei ist unter dem Begriff „Mindeststundenlohn“ jeweils ein Bruttostundenlohn ohne „Arbeitgeberbeiträge“ zu verstehen. Der GML wird schrittweise über $8,00 € \mathrm{ab}$

2 Da der Mindestlohn nur ex ante als Nominal größe festgesetzt werden kann, wirkt selbstverständlich die empirische "Lohnstückkostenelastizität des Konsumdeflators" auf die Reallohnausbeute der Mindestlohneinführung und in der Folge auf deren Beschäftigungs- und Wachstumseffekte.

3 Spezielle makroökonometrische Studien zu Mindestlohneffekten auf der Basis simulationsfähiger Kreislaufmodelle existieren nach meiner Kenntnis kaum. Interessanterweise bildet jedoch das makrökonometrische Modell der Bank of England (Bank of England 1999, S. 9f.) positive Beschäftigungseffekte $a b$.

4 Vgl. zur Grundstruktur des Modells und seiner Datenbasis: Bartsch 2002. Die verwendete Version LAPROSIM QD 10.32 enthält 548 Gleichungen, davon 118 Verhaltensgleichungen. 
dem 1.7.2008 und 8,50€ ab dem 1.1.2009 auf schließlich 9,00 € ab dem 1.7.2009 angehoben. Der Mindestlohn würde damit in etwa annähernd an das Niveau des „Spitzenreiters" Luxemburg angeglichen (Schulten 2006b, S. 76ff.). In den Folgejahren ab 2010 bis zum Ende des Simulationszeitraumes 2020 wird der Mindestlohn fortlaufend gemäß der „Meinhold-Formel“ (Preissteigerungsrate plus langfristiger Produktivitätstrend) angepasst.

Ein Mindestlohn von 7,50€ pro Stunde würde, ausgehend von einem Mittelwert der Stundenlöhne von etwa $6,00 €^{5} \mathrm{im}$ Niedriglohnsektor unterhalb dieses GMLStartniveaus, eine anfängliche Anhebung der Stundenlöhne für den Kreis der vom Startniveau begünstigten Beschäftigten von durchschnittlich $1,50 €$ implizieren.

Der Kreis der vom Anfangsniveau begünstigten Vollzeitarbeitskräfte wird auf 2,4 Mio. Personen geschätzt (Schulten 2005). Des Weiteren wird angenommen, dass sich die Zahl der bessergestellten geringfügig Beschäftigten und Teilzeitarbeitskräfte auf rund 3,1 Mio. beläuft. ${ }^{6}$ Durch die starke Steigerung des Mindestlohnsatzes bis Mitte 2009 und die anschließende Anpassung nach der „Meinhold-Formel“ wird der Kreis der Begünstigten langfristig bis auf knapp 4 Mio. Vollzeitbeschäftigte und 5,2 Mio. geringfügig und Teilzeitbeschäftigte erweitert. Auf der Grundlage der insgesamt uneindeutigen Tendenz der Ergebnisse der einschlägigen empirischen Panelstudien bezüglich der Beschäftigungswirkungen von Mindestlohneinführungen bzw. -erhöhungen im Niedriglohnsektor wird vorsichtig von einer Beschäftigungsneutralität in diesem Segment ausgegangen; d.h. der Kreis der unmittelbar GMLBegünstigten ist exogen gesetzt. Veränderungen der Beschäftigung insgesamt können daher im Kontext dieses Szenarios die Quote der Beschäftigten mit Mindestlohn verändern, nicht jedoch deren Zahl. Bezüglich der durch den GML begünstigten Mini- und Midijobber wird in diesem $\mathrm{Zu}-$ sammenhang vereinfachend angenommen, dass die Minderung ihres Arbeitsvolumens infolge der steigenden Löhne in etwa durch eine Ausweitung des Arbeitsvolumens von zu vollen Sätzen sozialversicherten, unfreiwillig Teilzeitbeschäftigten ausgeglichen wird.

Im Szenario wird dem Umstand Rechnung getragen, dass mit dem höheren Mindestlohn ein leichtes Absinken der durchschnittlichen Konsumquote und eine etwas höhere Steuerbelastung des „Durchschnittsbegünstigten“ einhergehen dürfte.

Die jährlichen Arbeitsvolumina der Niedriglöhner werden jeweils getrennt für die Gruppe der Vollzeitarbeitskräfte und die Gruppe der Teilzeitarbeitskräfte und geringfügig Beschäftigten modellendogen berechnet und anfänglich mit dem durchschnittlichen Anhebungsbetrag von 1,50€ multipliziert, um das durch den Mindestlohn induzierte zusätzliche Bruttolohnvolumen zu erhalten. Der im Szenario angenommene durchschnittliche Anhebungsbetrag erhöht sich mit jeder weiteren Anhebung des Mindestlohnes, allerdings nicht linear, sondern mit einem Abschlag, der dem steigenden Wert des Durchschnittslohnes der vom jeweiligen GMLNiveau Begünstigten Rechnung trägt. Bei der Kalkulation des durch den Mindestlohn ausgelösten Anstiegs der durchschnittlichen „Arbeitnehmerentgelte" wird zusätzlich der „Arbeitgeberbeitrag“ berücksichtigt.

Weiterhin wird beachtet, dass die Erhöhung der Bruttostundenlöhne der Vollzeitbeschäftigten auf 7,50 € im Einkommensteuergrundtarif $\mathrm{zu}$ - verglichen mit dem „Durchschnittslöhner“ - erheblich geringeren Steuerzahlungen führt. Die Gesamtbelastung des Bruttolohnes mit Steuern und Sozialabgaben wird mit etwa $25 \%$ angenommen (ohne Arbeitgeberbeiträge). Die „Nettolohnausbeute“ der Erhöhung niedriger Einkommen ist daher deutlich höher als im Bereich des Durchschnittslohnes.

Noch höher ist der durchschnittliche Nettolohnertrag für die Gruppe der geringfügig und Teilzeitbeschäftigten anzusetzen, da ein erheblicher Teil dieser Niedriglöhner Mini- und Midijobs mit reduzierter Steuer- und Sozialabgabenlast ausübt. Etwa $64 \%$ dieser Gruppe sind den geringfügig Beschäftigten zuzurechnen (Jaehrling et al. 2006). Da deren Pauschalabgaben im Regelfall vom Unternehmen getragen werden, ist der Bruttolohn für diese Beschäftigtengruppe gleich dem Nettolohn. ${ }^{7}$ Weitere 7 \% dieser Gruppe dürften zu den Midi-Jobbern zählen. ${ }^{8}$ Daher wird angenommen, dass die effektive durchschnittliche Belastung der Bruttolöhne der Gruppe der nicht Vollzeitbeschäftigten durch Steuern und Abgaben nur bei etwa der Hälfte jener der Vollzeitbeschäftigten liegt.

Zusätzlich wurde berücksichtigt, dass die Konsumquote des typischen Nied- riglöhners bei annähernd $100 \%$ und damit erheblich über dem Durchschnitt aller Einkommensbezieher liegen dürfte. ${ }^{9}$

\subsection{SIMULATIONSERGEBNISSE}

Der sogenannte „Kaitz-Index“, hier analog zur OECD-Definition gebildet als Relation des Mindestlohneinkommens bei Vollzeitbeschäftigung zum Durchschnittslohn bei Vollzeitbeschäftigung (jeweils in der Abgrenzung der „Arbeitnehmerentgelte“, also inklusive „Arbeitgeberbeiträge“), pendelt sich infolge der simulierten Werte für den gesetzlichen Mindestlohn im Schnitt auf einen Wert von etwa $40 \%$ und damit leicht oberhalb des Durchschnitts der OECDMindestlohnländer, aber noch deutlich unterhalb des Spitzenreiters Irland (über $50 \%$ ) ein. ${ }^{10} \mathrm{Da}$ die simulierten relativen Mindestlohnniveaus also keinen positiven „Ausreißer“ darstellen, sondern sich im Bereich der Spannweite empirisch beobachtbarer Kaitz-Werte für Länder mit GML - und damit auch im Rahmen des explizit oder implizit auf seine Beschäftigungswirkung hin untersuchten KaitzNiveaus - bewegen, kann erwartet werden, dass die modellexogen getroffenen Annahmen bezüglich der zu erwartenden $B e$ schäftigung zum Mindestlohn in Deutschland im Umsetzungsfall hinreichend realistisch sind.

Die Einführung eines Mindestlohnes von 7,50€ ab 2008 und die anschließenden deutlichen Steigerungen auf 9,00 $€$ im zweiten Halbjahr 2009 bewirken infolge

\footnotetext{
5 Auskunft Claus Schäfer, WSI Düsseldorf, dem ich für wichtige Anregungen und Hinweise für die Ausgestaltung des Szenarios danken möchte.

6 Dabei wird mangels besserer Daten die Annahme zugrundegelegt, dass die Relation von 3,9 Mio. geringfügig und in Teilzeitarbeitsverhältnissen Beschäftigten zu 3,0 Mio. Vollzeitbeschäftigten (1.3) in der Studie des IAT (Jaehrling et al. 2006) im Bereich der Niedriglöhner in etwa konstant ist.

7 Vgl. zur Abgabenbelastung der Mini- und Midijobber en détail: Brandt 2006.

8 Unter Anwendung der Relation von Midijobbern zu ausschließlich geringfügig Beschäftigten, vgl. dazu Bundesagentur 2004, S. 3.

9 Hinweise auf einen solchen Sachverhalt gibt die Wirtschaftsrechnung des Statistischen Bundesamts: Erst innerhalb der Haushaltsnettoeinkom mensgruppe von $2600 €$ bis unter $3600 €$ wird die durchschnittliche Sparquote von 11,3\% (2003) in etwa erreicht. Bis zu einem Haushaltsnettoeinkommen von $1300 €$ wird dagegen sogar entspart; vgl. Statistisches Bundesamt 2005: Tabellen 1.2.1 bis 1.2.13.

10 Vgl. Immervoll 2007, S. 9.
} 
Tabelle 1: Szenario gesetzlicher Mindestlohn: Der Beschäftigungseffekt und seine Hauptdeterminanten*

\begin{tabular}{|c|c|c|c|c|c|c|c|c|c|c|}
\hline & $\begin{array}{l}\text { Erwerbstätige } \\
\text { (in 1.000) }\end{array}$ & $\begin{array}{l}\text { Erwerbstätige } \\
\quad \text { (in \%) }\end{array}$ & $\begin{array}{l}\text { Reales BIP } \\
\text { (in \%) }\end{array}$ & $\begin{array}{l}\text { Privater } \\
\text { Konsum, } \\
\text { real } \\
(\text { in } \%)\end{array}$ & $\begin{array}{l}\text { Unternehmens- } \\
\text { investitionen } \\
\text { ohne Wohnbau, } \\
\text { real } \\
\text { (in \%) }\end{array}$ & $\begin{array}{l}\text { Erwerbstätigen- } \\
\text { produktivität } \\
\text { (in \%) }\end{array}$ & $\begin{array}{l}\text { Bruttosach- } \\
\text { kapital- } \\
\text { rendite } \\
\text { (in \%) }\end{array}$ & $\begin{array}{l}\text { Lohnquote } \\
\text { (in \%) }\end{array}$ & $\begin{array}{l}\text { Nettolohn- } \\
\text { summe } \\
\text { (in \%) }\end{array}$ & $\begin{array}{l}\text { Konsumpreis- } \\
\text { deflator } \\
\text { (in \%) }\end{array}$ \\
\hline 2007 & 0 & 0,00 & 0,00 & 0,00 & 0,00 & 0,00 & 0,00 & 0,00 & 0,00 & 0,00 \\
\hline 2008 & 315 & 0,81 & 0,46 & 0,73 & 0,73 & $-0,35$ & $-4,42$ & 1,18 & 2,85 & 0,04 \\
\hline 2009 & 463 & 1,20 & 0,89 & 1,28 & 2,07 & $-0,30$ & $-7,27$ & 2,11 & 5,73 & 0,45 \\
\hline 2010 & 317 & 0,82 & 0,93 & 1,28 & 2,40 & 0,11 & $-5,67$ & 1,91 & 6,20 & 1,18 \\
\hline 2011 & 94 & 0,24 & 0,69 & 1,02 & 1,27 & 0,45 & $-2,85$ & 1,20 & 5,16 & 1,76 \\
\hline 2012 & 19 & 0,05 & 0,51 & 0,88 & 0,23 & 0,46 & $-2,06$ & 0,91 & 4,64 & 1,94 \\
\hline 2013 & 43 & 0,11 & 0,36 & 0,80 & $-0,37$ & 0,25 & $-2,79$ & 0,98 & 4,68 & 1,90 \\
\hline 2014 & 100 & 0,26 & 0,30 & 0,89 & $-0,61$ & 0,04 & $-3,69$ & 1,18 & 5,06 & 1,85 \\
\hline 2015 & 165 & 0,44 & 0,39 & 1,14 & $-0,42$ & $-0,05$ & $-4,17$ & 1,38 & 5,72 & 1,89 \\
\hline 2016 & 220 & 0,59 & 0,55 & 1,40 & 0,00 & $-0,04$ & $-4,37$ & 1,53 & 6,47 & 2,00 \\
\hline 2017 & 239 & 0,64 & 0,66 & 1,55 & 0,28 & 0,02 & $-4,41$ & 1,64 & 7,04 & 2,16 \\
\hline 2018 & 211 & 0,57 & 0,69 & 1,57 & 0,29 & 0,12 & $-4,34$ & 1,69 & 7,33 & 2,34 \\
\hline 2019 & 162 & 0,44 & 0,68 & 1,55 & 0,20 & 0,24 & $-4,25$ & 1,72 & 7,48 & 2,51 \\
\hline 2020 & 121 & 0,33 & 0,69 & 1,57 & 0,19 & 0,36 & $-4,35$ & 1,79 & 7,70 & 2,65 \\
\hline
\end{tabular}

der überwiegenden Begünstigung von Haushalten mit deutlich unterdurchschnittlicher Steuerbelastung eine erhebliche Steigerung der Nettoarbeitsentgelte der Lohnabhängigen. Der hohe Anteil von geringfügig Beschäftigten, bei denen Bruttound Nettolohn in der Regel zusammenfällt, und Midijobbern mit reduzierten Sozialversicherungssätzen im Niedriglohnbereich erhöht die "Nettolohnausbeute“ der Mindestlohneinführung zusätzlich. Die Anhebung des Mindestlohnes auf 7,75 € im Schnitt des Jahres 2008 entspricht einer gesamtwirtschaftlichen Anhebung des durchschnittlichen „Arbeitnehmerentgelts“ um etwa $1,5 \%$. Die Fokussierung der Anhebung auf den Niedriglohnsektor führt zu einer Anhebung des Nettoreallohnes um $1,8 \%$. Der begünstigte Personenkreis ist zusätzlich durch eine im Schnitt weit überdurchschnittliche Konsumquote gekennzeichnet. Das Zusammenwirken von hohem Nettolohneffekt und hoher Konsumquote löst einen beachtlichen Konsumschub aus: Bereits in 2008 erhöht sich der private Konsum um 0,7 \% gegenüber dem Basispfad (Abbildung 1; Tabelle 1). Die deutliche und nachhaltig positive Erhöhung des realen privaten Konsums regt zusätzliche Anlageinvestitionen des Unternehmenssektors zwecks Anpassung des Kapitalstocks an das höhere Nachfrageniveau an. Beide Komponenten gemeinsam erhöhen die Binnennachfrage und mit ihr auch das reale Bruttoinlandsprodukt insgesamt erheblich und führen in der kurzen Frist (2008) zu einer Erhöhung des Beschäftigungsniveaus um über 300.000 Personen (Abbildung 2; Tabelle 1). Das Be-

Abb. 1: Auswirkungen eines gesetzlichen Mindestlohns auf die Verwendungsaggregate* - in \% -

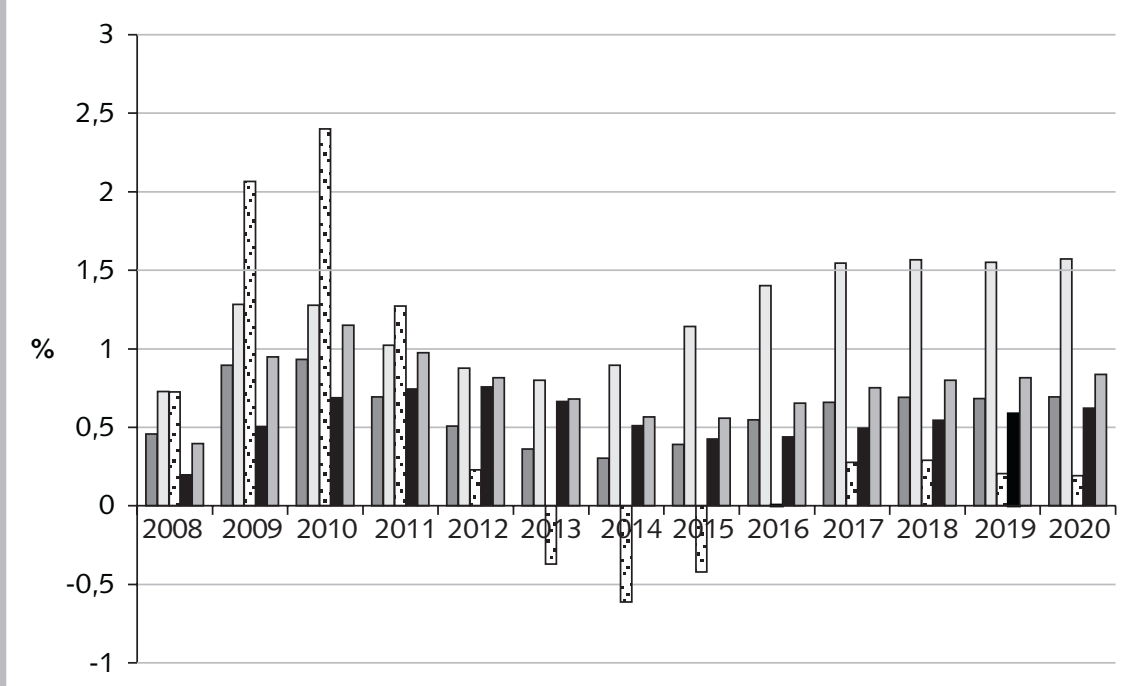

\begin{tabular}{|l|}
\hline Reales Bruttoinlandsprodukt \\
$\square$ Privater Konsum, real \\
$\square$ Bruttoinvestitionen der Unternehmen ohne Wohnbau, real \\
$\square$ Exportvolumen \\
$\square$ Importvolumen \\
\hline
\end{tabular}

*Dargestellt sind die prozentualen Abweichungen vom Basisszenario (vgl. Abschnitt 3.2).

Quelle: Berechnungen und Darstellung des Autors.

WSI MitTeILUNGeN

schäftigungsmaximum wird in 2009 mit etwa 450.000 zusätzlich Erwerbstätigen erreicht.

Außenwirtschaftlich ist die Einführung eines GML unschädlich. ${ }^{11}$ Die positiven „Feedbackeffekte“ der steigenden deutschen Importnachfrage, die sich, vermittelt über ein stärkeres Wachstum der eng verzahnten übrigen Europäischen Union
(EU), als eine verstärkte Nachfrage nach deutschen Exporten manifestieren, übertreffen quantitativ die gegenläufigen Wirkungen der eher bescheidenen Erhöhung

11 Der EU-Raum wird über ein kleines, mit dem Deutschlandmodell interdependent verknüpftes „nutshell model“ abgebildet. 


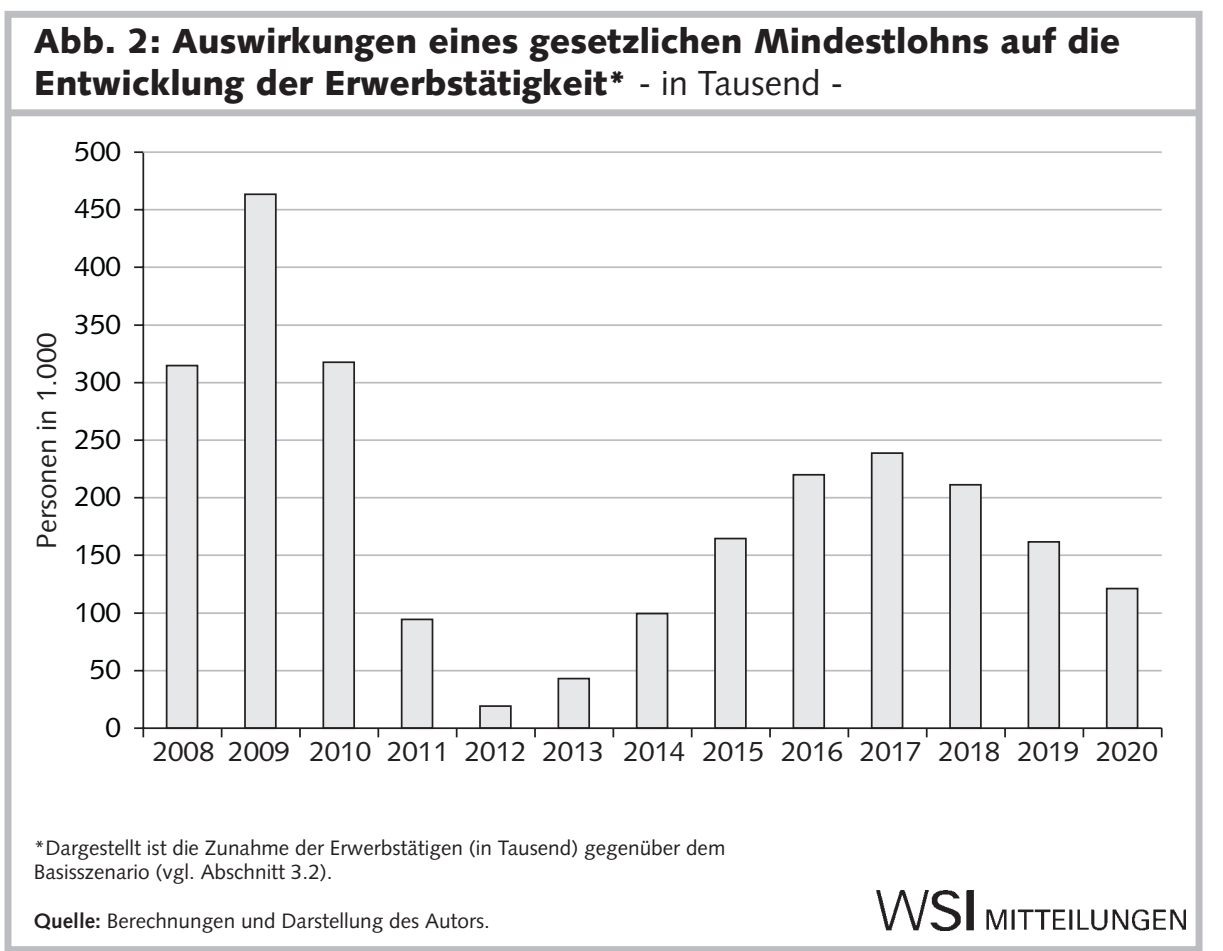

\section{Abb. 3: Zentrale Bestimmungsgrößen des Beschäftigungseffekts* nach Einführung eines gesetzlichen Mindestlohns}

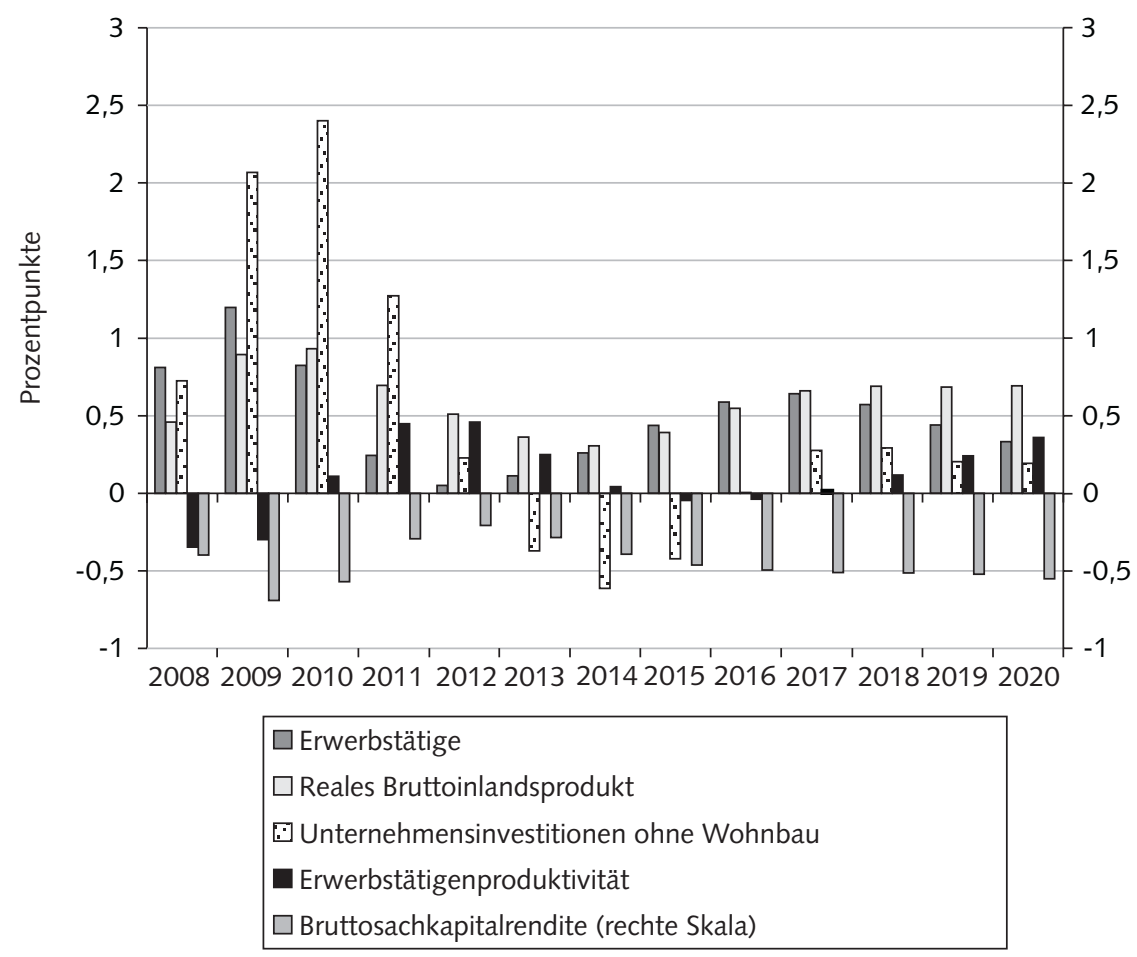

*Dargestellt sind die Abweichungen gegenüber dem Basisszenario (vgl. Abschnitt 3.2).

Quelle: Berechnungen und Darstellung des Autors.

der Commodity Terms of Trade, ${ }^{12}$ welche aus den moderaten, GML-bedingten Lohnstückkostenerhöhungen resultiert, zumal das induzierte leicht höhere Wachstum in der „Rest-EU“ auch dort zu einer - sehr geringfügigen - Mehrinflation führt.
WSI MITTELUNGEN

schuss über den gesamten Simulationszeitraum betrachtet insgesamt leicht ab.

In den Folgejahren reduziert sich der anfängliche Beschäftigungsgewinn wieder. Hierfür sind vor allem drei Faktoren ursächlich:

(1) Der jeweils zweistellige Anstieg der Löhne im Bereich des GML in der „Startphase" 2008/09 schwächt sich ab 2010 deutlich auf Wachstumsraten im Bereich von $4 \%$ per anno ab, wodurch sich das GML-induzierte zusätzliche nominale Konsumwachstum vermindert.

(2) Die (moderaten) Preiswirkungen der GML-induzierten deutlichen Lohnsteigerungen in 2008/09 höhlen den anfänglichen Nettoreallohneffekt zeitverzögert in den Jahren 2009/11 graduell aus (Tabelle 1). Dadurch vermindert sich die Differenz des realen Konsums gegenüber dem Referenzszenario bis zum Ende des Simulationszeitraumes in 2020 allmählich auf etwa die Hälfte des Wertes im angenommenen Jahr der Mindestlohneinführung 2008.

(3) Das insgesamt höhere Anlageinvestitionsniveau der Unternehmen hat eine erhöhte Diffusion des technischen Fortschritts in den Kapitalstock zur Folge (,embodied technical progress“), wodurch die Arbeitsproduktivität je Erwerbstätigen nachhaltig erhöht wird (Abbildung 3; Tabelle 1). Zugleich erzeugen die infolge des Mindestlohnes steigenden nominalen und realen Lohnstückkosten (Tabelle 1) zusätzliche Rationalisierungsanreize („Ex-AnteFaktorsubstitution"), welche die Arbeitsproduktivität zusätzlich steigern.

Insbesondere aufgrund des überproportionalen Wachstums des realen privaten Konsums gegenüber der realen Gesamtnachfrage (Abbildung 1) bei gleichzeitig überdurchschnittlicher Arbeitsintensität und überdurchschnittlichem "local content" (respektive unterdurchschnittlicher Importquote) der Produktion von Konsumgütern und Dienstleistungen bleiben jedoch auch mittelfristig geringfügige positive Beschäftigungseffekte erhalten (Abbildung 1).

Der prognostizierten leichten Steigerung des Exportvolumens steht infolge der vor allem die Binnennachfrage belebenden Wirkung des GML eine stärkere Steigerung des Importvolumens gegenüber (Abbildung 1). Im Ergebnis sinkt der Exportüber- 
Langfristig steigt die GML-induzierte Zusatzbeschäftigung wieder leicht an. Für diese Entwicklung ist im Kern die weitere Investitionsdynamik ursächlich (Abbildung 3): Kurzfristig dominiert in der Investitionsfunktion zwar der Einfluss der stark wachsenden zusätzlichen Nachfrage; mittelfristig wird jedoch der Effekt der infolge des GML leicht sinkenden Rendite auf das Investitionsniveau spürbar. Wie bereits erwähnt, schwächt sich zugleich ab 2010 auch der GML-induzierte Konsumnachfrageimpuls infolge sinkender GML-Zuwachsraten ab. Durch das sinkende Investitionsniveau verlangsamt sich die Entwicklung der Arbeitsproduktivität relativ zum Basisszenario, sodass sich langfristig wieder der Effekt der insgesamt nachhaltig gestiegenen Konsumnachfrage auf das Beschäftigungsniveau durchsetzen kann. Infolge dieses Prozesses pendelt sich das Investitionsniveau langfristig etwas oberhalb des Niveaus der Basislösung ein. Auf eine durch den anfänglichen kräftigen "Nachfrageschock“ ausgelöste Anfangsschwingung von Wachstum und Beschäftigung mit stark ausgeprägter Amplitude folgt mittel- bis langfristig eine gedämpfte und gestreckte Schwingung. Langfristig bleiben Mehrbeschäftigungseffekte von über 100.000 Personen erhalten (Abbildung 2; Tabelle 1).

Steigende Beschäftigung bei insgesamt steigendem Nominallohnniveau verbessert in der Gesamtbetrachtung die Einnahmen-/Ausgabenrelation des gesetzlichen Sozialversicherungssystems und erlaubt Senkungen des aggregierten Sozialversicherungssatzes gegenüber dem Referenzszenario, deren Niveau, wenig erstaunlich, zeitverzögert in etwa der prognostizierten mindestlohnbedingten Beschäftigungsänderung folgt (Tabelle 2). Auch der Staat profitiert über den gesamten Zeitraum; dabei folgt das jeweilige Niveau der Verbesserung des Finanzierungssaldos des Staates relativ zum BIP („Defizitquote“) der Entwicklung der Niveaus von Wachstum und Beschäftigung. Insgesamt verbessert sich

\begin{tabular}{|c|c|c|c|}
\hline & $\begin{array}{c}\text { Aggregierter } \\
\text { Sozialversicherungssatz } \\
\text { (in Prozentpunkten) }\end{array}$ & $\begin{array}{l}\text { Defizitquote } \\
\text { (in Prozentpunkten; } \\
\text { positiver Wert } \\
=\text { Verbesserung) }\end{array}$ & $\begin{array}{l}\text { Staatsschuldenquote } \\
\text { (in Prozentpunkten) }\end{array}$ \\
\hline 2007 & 0,00 & 0,00 & 0,00 \\
\hline 2008 & $-0,21$ & 0,44 & $-0,50$ \\
\hline 2009 & $-0,57$ & 0,70 & $-1,44$ \\
\hline 2010 & $-0,52$ & 0,53 & $-2,28$ \\
\hline 2011 & $-0,20$ & 0,21 & $-2,61$ \\
\hline 2012 & $-0,04$ & 0,10 & $-2,64$ \\
\hline 2013 & $-0,06$ & 0,11 & $-2,60$ \\
\hline 2014 & $-0,09$ & 0,19 & $-2,64$ \\
\hline 2015 & $-0,17$ & 0,30 & $-2,81$ \\
\hline 2016 & $-0,26$ & 0,38 & $-3,09$ \\
\hline 2017 & $-0,30$ & 0,43 & $-3,39$ \\
\hline 2018 & $-0,25$ & 0,45 & $-3,68$ \\
\hline 2019 & $-0,14$ & 0,45 & $-3,93$ \\
\hline 2020 & $-0,05$ & 0,49 & $-4,16$ \\
\hline
\end{tabular}

infolge dieser Entwicklung des öffentlichen Finanzierungssaldos auch die Relation der Staatsverschuldung zum Bruttoinlandsprodukt (Tabelle 2).

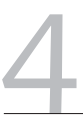

\section{Resümee}

Als Ergebnis einer aktualisierten Simulationsstudie auf der Basis eines Mindestlohnszenarios, das dem Konzept der Dienstleistungsgewerkschaft ver.di zur Einführung eines gesetzlichen Mindestlohns ab 2008 folgt, lässt sich festhalten, dass die Einführung eines Mindestlohnes in Deutschland kurz- bis mittelfristig die Binnennachfrage infolge der überdurchschnittlich hohen Konsumquote bei zugleich unterdurchschnittlicher Steuer- und Abgabenbelastung der Niedriglohnempfänger spürbar anregen dürfte. Dieser Nachfrageschub bewirkt, ebenfalls auf kurze bis mittlere Sicht, nennenswerte Beschäftigungseffekte von maximal etwa 450.000 Beschäftigten. Langfristig bilden sich die anfänglichen
Wachstums- und Beschäftigungswirkungen aufgrund zeitverzögert wirksamer Preis- und Rationalisierungseffekte partiell wieder zurück. Bei einer schrittweisen Anhebung des Mindestlohnes auf 9,00€ bis Mitte 2009 und anschließender Indexierung mit der „Meinhold-Formel“ bleiben jedoch leichte, aber dauerhafte Beschäftigungsgewinne in Höhe von über 100.000 Personen gegenüber dem Fall des Verzichts auf die Einführung eines Mindestlohnes erhalten.

Insgesamt lässt sich feststellen, dass sich nach der Prognose des Modells durch die Einführung eines Mindestlohnes die materiellen Lebensgrundlagen einiger Millionen zurzeit prekär niedrig entlohnter Beschäftigter verbessern lassen, ohne dass, wie von den zahlreichen Vertretern des einfachen neoklassischen Arbeitsmarktmodells in Deutschland behauptet, Beschäftigungsverluste zu erwarten wären im Gegenteil. Dieses Ergebnis ist durchaus kompatibel mit den Befundenen etlicher renommierter partialanalytischer empirischer Studien zum Zusammenhang von Mindestlöhnen und Beschäftigung. 
Artus, I./Schmidt, R./Sterkel, G. (2000): Brüchige Tarifrealität, Berlin 2000

Bank of England (1999): Economic models at the Bank of England, London

Bartsch, K. (2002): Das makroökonometrische Deutschlandmodell LAPROSIM QD 8.3 E, WSI Diskussionspapier 108, Düsseldorf Bhaskar, V./To, T. (1999): Minimum Wages for Ronald McDonald Monopsonies - A Theorie of Monopsonic Competition, in: Economic Journal, April, S. 190-203

Bispinck, R./Schäfer, C. (2006): Niedriglöhne und Mindesteinkommen in Deutschland, in: Schulten, T./Bispinck, R./Schäfer, C. (Hrsg): Mindestlöhne in Europa, Hamburg, S. 269-297

Bosch, G./Weinkopf, C. (2006): Mindestlöhne - eine Strategie gegen Lohn- und Sozialdumping ?, in: Wirtschafts- und sozialpolitisches Forschungs- und Beratungszentrum der Friedrich-Ebert-Stiftung, Abteilung Arbeit und Sozialpolitik, Gesprächskreis Sozialpolitik, Referat internationale Politikanalyse: Sozialer Ausgleich in den alten und neuen Mitgliedsländern der EU: Tagung des Steuerungskreises „Europäisches Wirtschaftsund Sozialmodell“ der Friedrich-Ebert-Stiftung, Dokumentation, Bonn 2006, S. 26-35

Brandt, T. (2006): Bilanz der Minijobs und Reformperspektiven, in: WSIMitteilungen 8, S. 446-452

Bundesagentur für Arbeit (2004): Mini- und Midijobs in Deutschland, Sonderbericht, Nürnberg

Card, D./Krueger, A. (1994): Minimum Wages and Employment: A Case Study of the Fast-Food Industry in New Jersey and Pennsylvania, American Economic Review 5

Deutsches Institut für Wirtschaftsforschung (DIW) (2006): Wachsender Niedriglohnsektor in Deutschland - Sind Mindestlöhne sinnvoll?, DIWWochenbericht 15-16

Dolado, J./Kramarz, F./Machin, S./Manning, A./Margolis, D./Teulings, C. (1996): The economic impact of minimum wages in Europe, "Economic Policy" , October

Draca, M./Machin, S./Van Reenen, J. (2006): Minimum wages and Firm Profitability, IZA Discussion Paper 1913, Bonn

Herr, H. (2002): Wages, Employment and Prices, Business Institute Berlin at the FHW Berlin - Berlin School of Economics Paper 15, Berlin Institut für Makroökonomie und Konjunkturforschung (IMK) (2006): Wirtschaftliche Entwicklung 2006 und 2007, IMK-Report, April Immervoll, H. (2007): Minimum Wages, Minimum Labour Costs and the Tax Treatment of Low-Wage Employment, IZA Discussion Paper 2555, Bonn

Jaehrling, K./Kalina,T./Vanselow, A./Voss-Dahm, D. (2006): Niedriglohnarbeit in der Praxis - Arbeit in Häppchen für wenig Geld, in: Sterkel, G./Schulten, T./Wiedemuth, J. (Hrsg.): Mindestlöhne gegen Lohndumping, Hamburg; S. 114-141

Kalina, T./Weinkopf, C. (2006a): Mindestens sechs Millionen Niedriglohnbeschäftigte in Deutschland: Welche Rolle spielen Teilzeitbeschäftigte und Minijobs ?, IAT-Report 2006-03, Gelsenkirchen
Kalina, T./Weinkopf, C. (2006b): Ein gesetzlicher Mindestlohn auch in Deutschland?, IAT-Report 2006-06, Gelsenkirchen

Klauder, W./Schnur, P./Zika, G. (1996): Wege zu mehr Beschäftigung Simulationsrechnungen bis zum Jahr 2005 am Beispiel Westdeutschland, IAB Werkstattbericht 5, Nürnberg

Kromphardt, J. (1987): Arbeitslosigkeit und Inflation, Göttingen

Kyi, M./Neuhaus, R./Wenke, M. (1991): Simulationen mit ökonometrischen Modellen, in: Rheinisch-Westfälisches Institut für Wirtschaftsforschung (Hrsg.): Arbeit mit ökonometrischen Modellen, Essen

Mankiw, G. (1996): Makroökonomik, Wiesbaden

Marx, K. (1974): Das Kapital, Bd. 1, Berlin

OECD (1998): OECD Employment Outlook, Paris

Neumark, D./Wascher, W. (2007): Minimum Wages and Employment, IZA Discussion Paper 2570, Bonn

Ragacs, C. (2003): Mindestlöhne und Beschäftigung: Ein Überblick über die neuere empirische Literatur, Wirtschaftsuniversität Wien, Working Paper Series: Growth and Employment in Europe: Sustainability and Competitivness, Working Paper 25, Wien

Ragnitz, J./Thum, M. (2007): Zur Einführung von Mindestlöhnen: Empirische Relevanz des Niedriglohnsektors, Mimeo, Halle/Dresden Rhein, T./Stamm, M. (2006): Niedriglohnbeschäftigung in Deutschland, IAB Forschungsbericht 12, Nürnberg

Sachverständigenrat (SVR) (2004): Jahresgutachten 2004/05 des Sachverständigenrates zur Begutachtung der gesamtwirtschaftlichen Entwicklung

Sachverständigenrat (SVR) (2005): Jahresgutachten 2005/06 des Sachverständigenrates zur Begutachtung der gesamtwirtschaftlichen Entwicklung

Sachverständigenrat (SVR) (2006): Jahresgutachten 2006/07 des Sachverständigenrates zur Begutachtung der gesamtwirtschaftlichen Entwicklung

Schulten, T. (2005): Politische Ökonomie gesetzlicher Mindestlöhne, in: Hein, E./Heise, A./Truger, A. (Hrsg.): Löhne, Beschäftigung, Verteilung und Wachstum, Marburg

Schulten, T. (2006a): Gesetzliche und tarifliche Mindestlöhne in Europa ein internationaler Überblick, in: Schulten, T./Bispinck, R./Schäfer, C. (Hrsg.): Mindestlöhne in Europa, Hamburg, S. 9-27

Schulten, T. (2006b): Mindestlöhne in den BeNeLux-Staaten, in: Schulten, T./Bispinck, R./Schäfer, C. (Hrsg.): Mindestlöhne in Europa, Hamburg, S. 71-101

Sinn, H.-W. (2004): Ist Deutschland noch zu retten?, Berlin Statistisches Bundesamt (2000): Volkswirtschaftliche Gesamtrechnungen, Fachserie 18, Reihe 2 Input-Output-Rechnung 1995, Wiesbaden Statistisches Bundesamt (2005): Fachserie 15, Heft 4, Wirtschaftsrechnung - Einkommens- und Verbrauchsstichprobe - Einnahmen und Ausgaben privater Haushalte 2003, Wiesbaden

Stigler, G. (1946): The Economics of Minimum Wage Legislation, American Economic Review 3, S. 358-365

Walter, H. (1983): Wachstums- und Entwicklungstheorie, Stuttgart 